\title{
Supplementary materials of
}

\section{Oxidative capacity and radical chemistry in the polluted atmosphere of Hong Kong and}

Pearl River Delta region: analysis of a severe photochemical smog episode

Likun Xue ${ }^{1 *}$, Rongrong Gu${ }^{1}$, Tao Wang ${ }^{2,1}$, Xinfeng Wang ${ }^{1}$, Sandra Saunders ${ }^{3}$, Donald Blake ${ }^{4}$, Peter K. K. Louie ${ }^{5}$, Connie W. Y. Luk ${ }^{5}$, Isobel Simpson ${ }^{4}$, Zheng Xu ${ }^{1}$, Zhe Wang ${ }^{2}$, Yuan Gao ${ }^{2}$, Shuncheng Lee ${ }^{2}$, Abdelwahid Mellouki ${ }^{1}$, and Wenxing Wang ${ }^{1}$

${ }^{1}$ Environment Research Institute, Shandong University, Ji’nan, Shandong, China

${ }^{2}$ Department of Civil and Environmental Engineering, Hong Kong Polytechnic University, Hong Kong, China

${ }^{3}$ School of Chemistry and Biochemistry, University of Western Australia, WA, Australia

${ }^{4}$ Department of Chemistry, University of California at Irvine, Irvine, CA, USA

${ }^{5}$ Environmental Protection Department, the Government of Hong Kong Special Administrative Region, Hong Kong, China

*To whom correspondence should be addressed

E-mail:xuelikun@sdu.edu.cn, Tel: +86-531-88361185

Page $2 \quad$ Table S1

Page 3 Figure $S 1$

Page 4 Figure $S 2$

Page $5 \quad$ Figures $S 3$ and S4

Page 6 Figure S5

Page $7 \quad$ Figure 56 
Table S1. Summary of field measurements at Tung Chung in summer 2011

\begin{tabular}{|c|c|c|}
\hline Species & Instrument or techniques & Time resolution \\
\hline $\mathrm{O}_{3}$ & TEI 49i & $1 \mathrm{~min}$ \\
\hline $\mathrm{CO}$ & API 300EU & $1 \mathrm{~min}$ \\
\hline $\mathrm{SO}_{2}$ & $T E I 43 i$ & $1 \mathrm{~min}$ \\
\hline $\mathrm{NO} \& \mathrm{NO}_{2}$ & TEI $42 i+$ blue light converter & $1 \mathrm{~min}$ \\
\hline NO \& NOy & TEI $42 c y+\mathrm{MoO}$ converter & $1 \mathrm{~min}$ \\
\hline HONO & LOPAP & $1 \mathrm{~min}$ \\
\hline $\mathrm{ClNO}_{2}$ & CIMS & $6 \mathrm{sec}$ \\
\hline PANs & CIMS & $6 \mathrm{sec}$ \\
\hline $\mathrm{H}_{2} \mathrm{O}_{2}$ \& organic peroxides & Aerolaser AL-2021 & $1 \mathrm{~min}$ \\
\hline $\mathrm{C}_{1}-\mathrm{C}_{10}$ hydrocarbons & Canister + GC/FID/ECD/MS & 24-hour \\
\hline $\mathrm{C}_{2}-\mathrm{C}_{10}$ hydrocarbons & $\begin{array}{l}\text { Syntech Spectras, model GC } 955 \\
\text { Series 600/800 POСP }\end{array}$ & $30 \mathrm{~min}$ \\
\hline $\mathrm{C}_{1}-\mathrm{C}_{8}$ carbonyls & $\begin{array}{l}\text { DNPH-coated sorbent cartridge } \\
\text { sampling }+H P L C\end{array}$ & $\begin{array}{l}\text { 24-hour in general; } \\
\text { 3-hour on episode }\end{array}$ \\
\hline $\mathrm{PM}_{2.5} \& \mathrm{PM}_{10}$ mass & SHARP & $1 \mathrm{~min}$ \\
\hline $\begin{array}{l}\mathrm{SO}_{4}^{2-}, \mathrm{NO}_{3}^{-}, \mathrm{NH}_{4}^{+}, \mathrm{Cl}^{-}, \mathrm{Na}^{+}, \mathrm{Ca}^{2+} \\
\mathrm{K}^{+} \text {in } \mathrm{PM}_{2.5}\end{array}$ & $M A R G A$ & 1 hour \\
\hline $\mathrm{OC} \& \mathrm{EC}$ in $\mathrm{PM}_{2.5}$ & Sunset OCEC analyzer & 1 hour \\
\hline $\mathrm{BC}$ in $\mathrm{PM}_{2.5}$ & Magee & $5 \min$ \\
\hline Aerosol scattering coefficient & Ecotech Nephelometer & $1 \mathrm{~min}$ \\
\hline $\begin{array}{l}\text { Particle number concentration } \\
(5 \mathrm{~nm}-10 \mu \mathrm{m})\end{array}$ & MSP/WPS Model 1000XP & $8 \min$ \\
\hline$J_{\mathrm{NO} 2}$ & Metcon Filter Radiometer & $5 \mathrm{sec}$ \\
\hline Temperature \& RH & Young $R H / T$ probe & $5 \mathrm{sec}$ \\
\hline Wind speed and direction & Gill WindSonic & $5 \mathrm{sec}$ \\
\hline Solar Radiation & LI-200 Pyranometer Sensor & $5 \mathrm{sec}$ \\
\hline
\end{tabular}




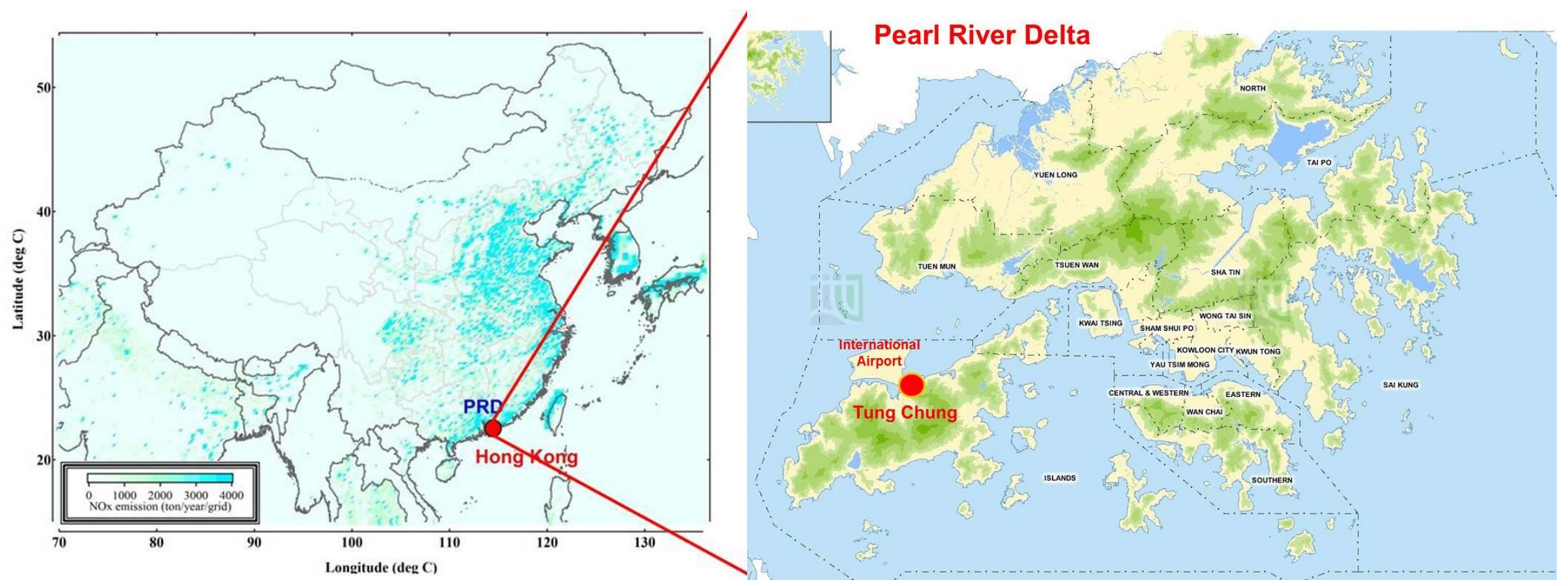

Figure S1. Map showing the locations of Hong Kong, the Pearl River Delta region and the study site at Tung Chung (TC). 

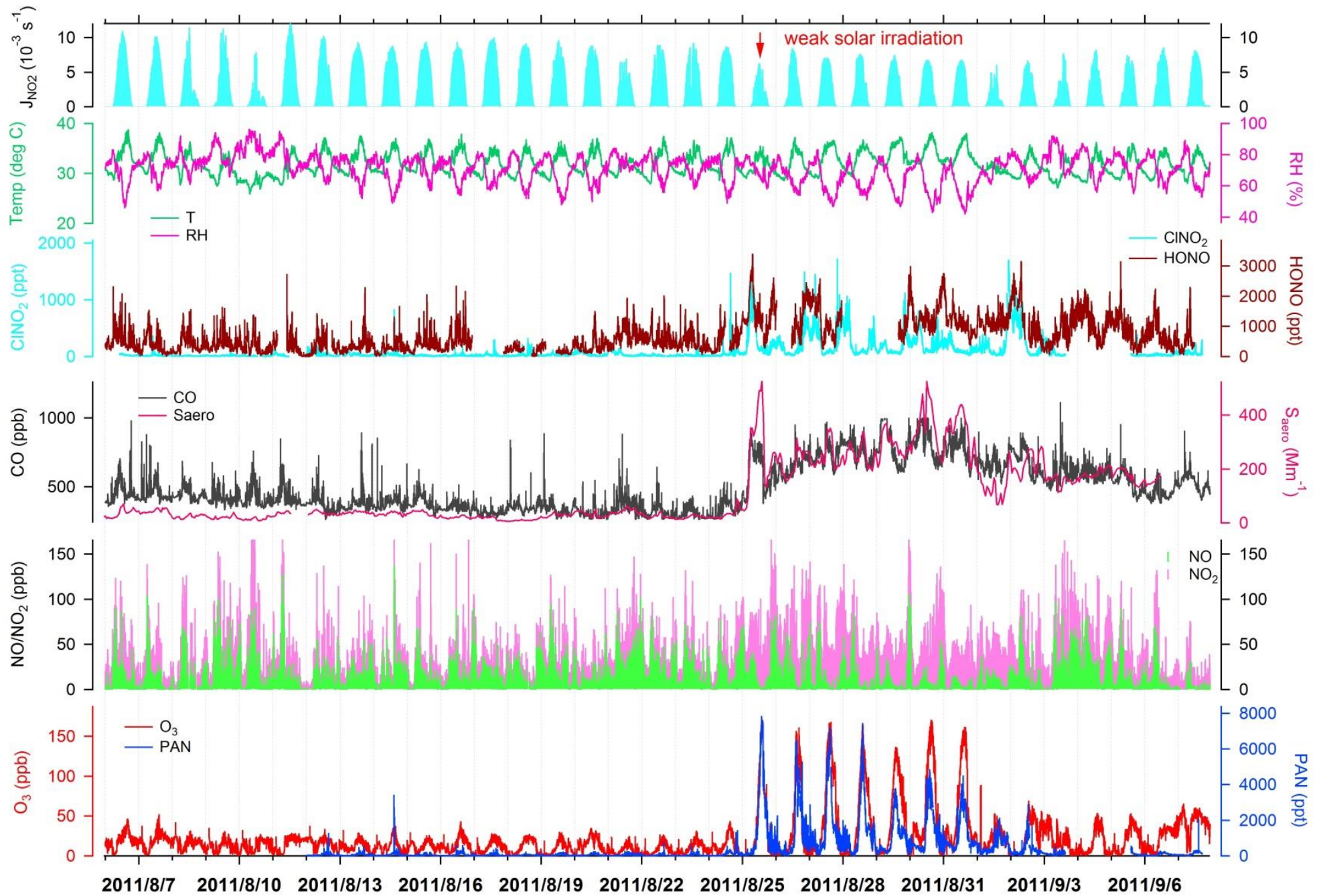

Figure S2. Time series of air pollutants and meteorological parameters observed at Tung Chung during the intensive campaign from 6 August to 7 September 2011. $S_{\text {aero }}$ stands for the aerosol scattering coefficient of $\mathrm{PM}_{2.5}$. 

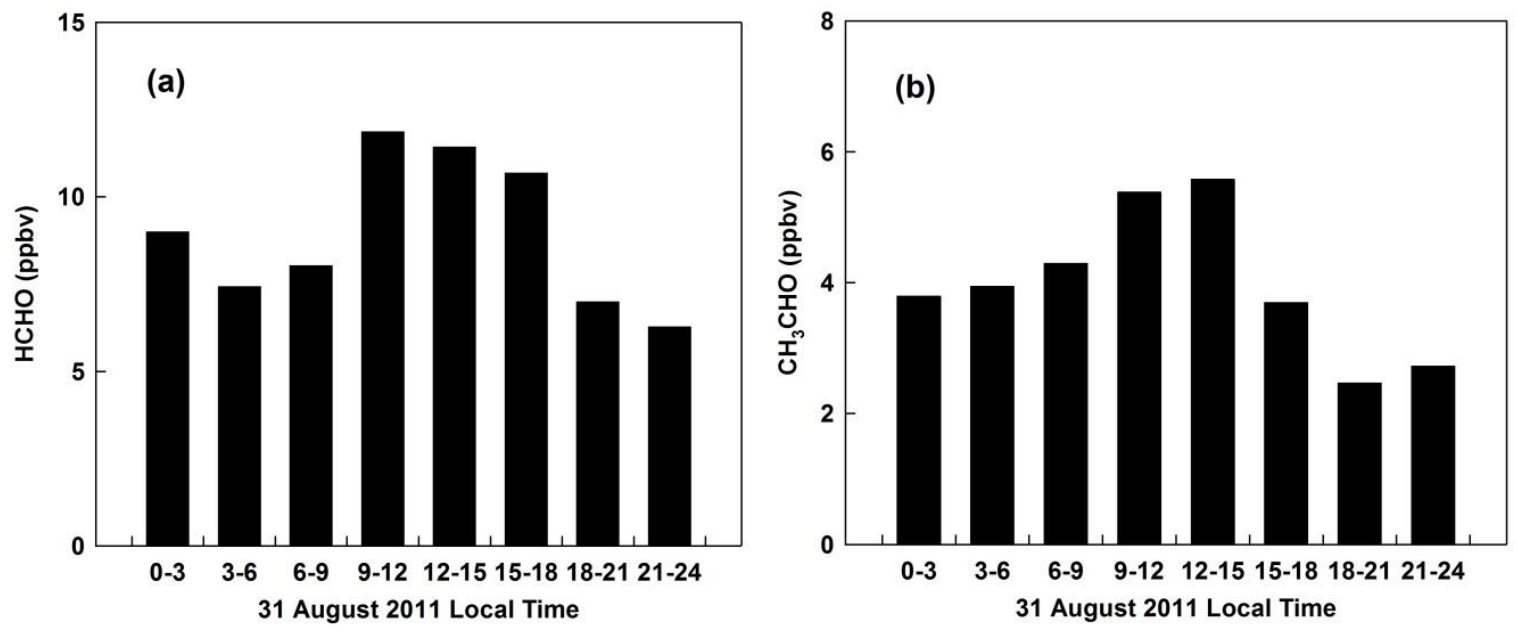

Figure S3. Diurnal variations of (a) formaldehyde and (b) acetaldehyde measured at Tung Chung on 31 August 2011.

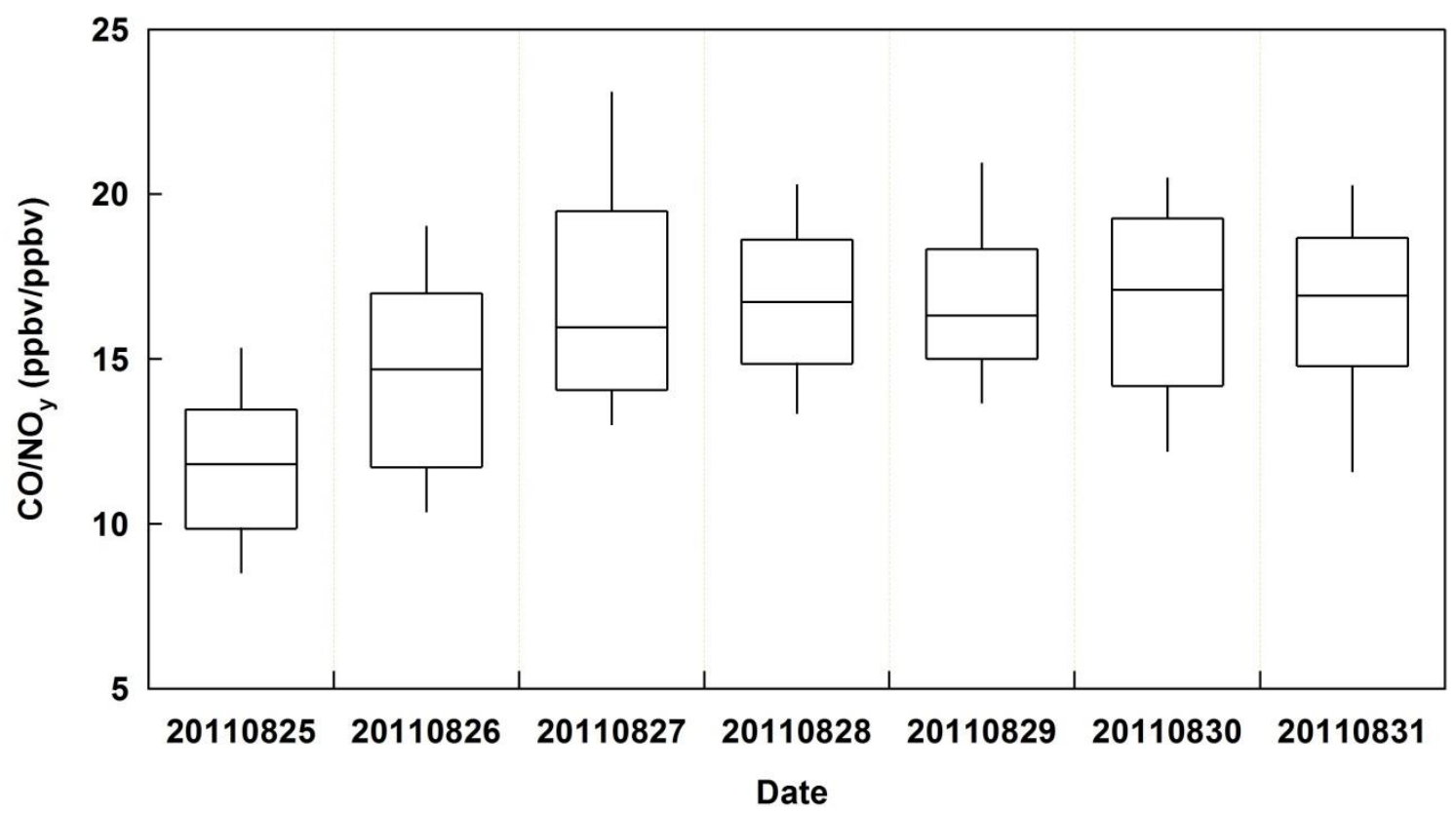

Figure S4. Distributions of the daytime (08:00-18:00 local time) $\mathrm{CO} / \mathrm{NO}_{\mathrm{y}}$ ratios measured at Tung Chung from 25-31 August 2011. The whisker plot provides the $90^{\text {th }}, 75^{\text {th }}, 50^{\text {th }}, 25^{\text {th }}$ and $10^{\text {th }}$ percentiles of the measurement data. 

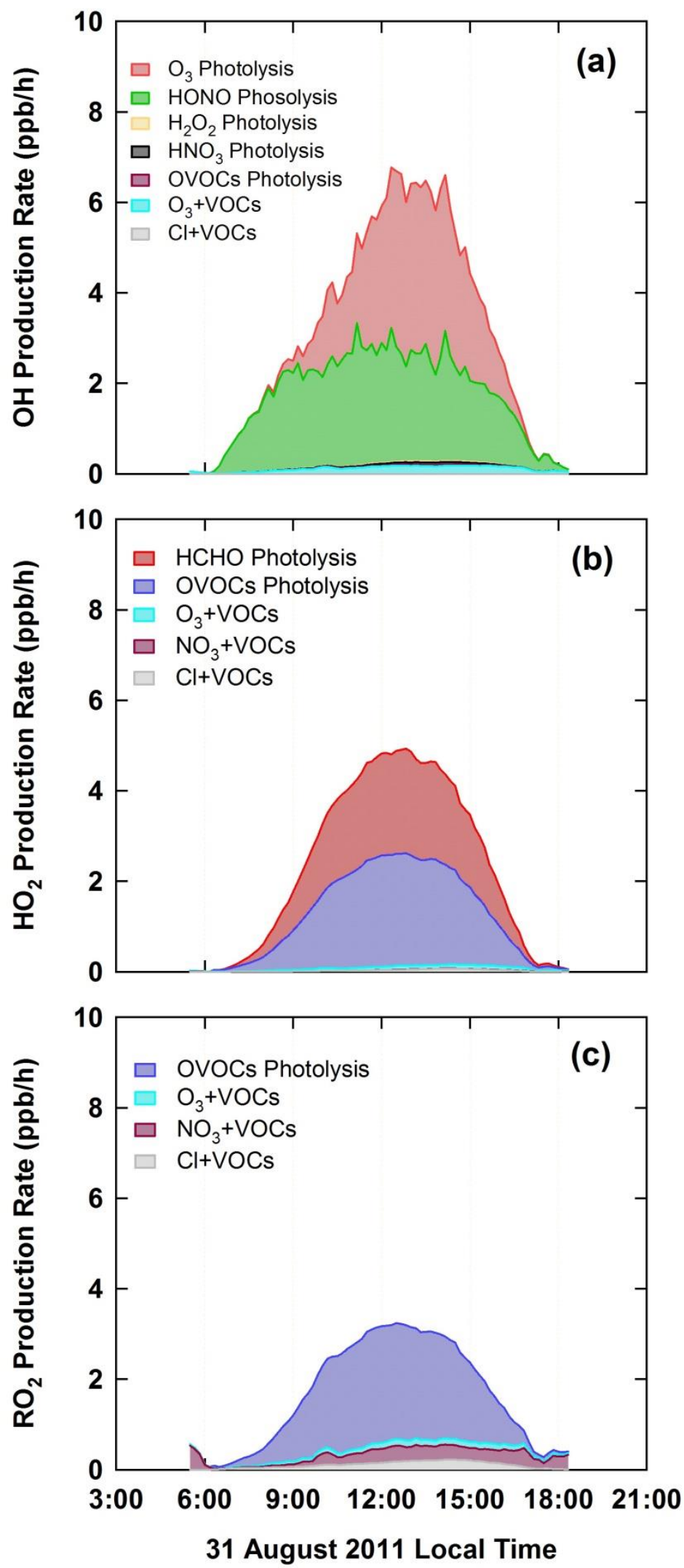

Figure S5. Primary daytime sources of (a) $\mathrm{OH}$, (b) $\mathrm{HO}_{2}$ and (c) $\mathrm{RO}_{2}$ radicals at Tung Chung on 31 August 2011. 


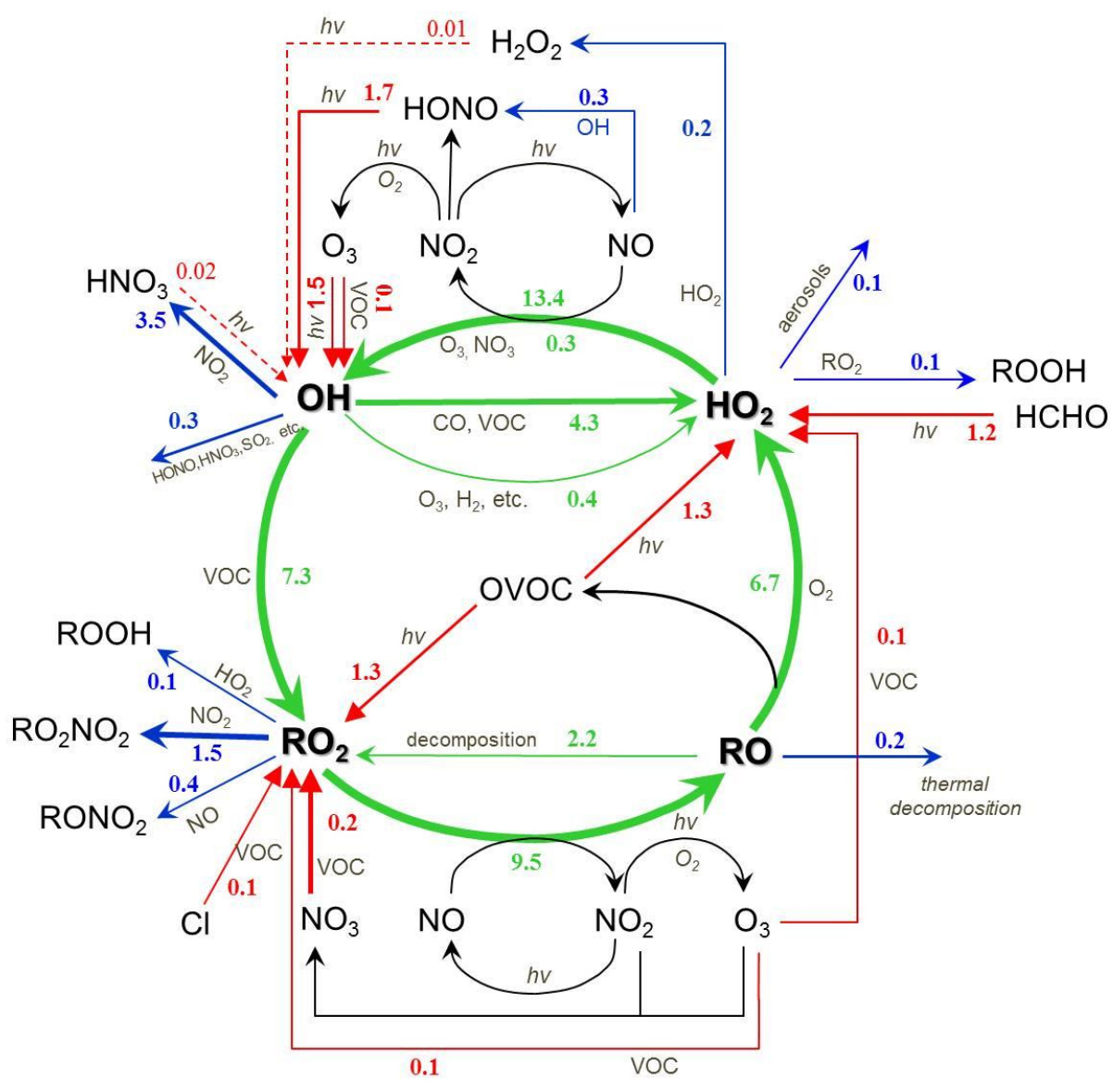

Figure S6. Daytime average $\mathrm{RO}_{\mathrm{X}}$ budget at Tung Chung on 31 August 2011. The units are $\mathrm{ppb} / \mathrm{h}$. The red, blue and green lines indicate the production, destruction and recycling pathways of radicals, respectively. 